\title{
CORRESPONDENCE \\ ETIOLOGICAL TREATMENT OF CHRONIC CHAGAS DISEASE
}

Re: "Persistent infections in chronic Chagas'disease patients treated with anti-Trypanosoma cruzi nitroderivatives"

Belo Horizonte, 08 February 2001

Sir,

During the last thirty years there has been a controversy concerning the etiological treatment of chronic Chagas disease, chiefly because there is no consensus for the criterion of cure.

The article recently published in this Journal ${ }^{1}$ merits the attention of the community interested in the subject. The authors' summary is as follows:

SUMMARY. We used a molecular method and demonstrated that treatment of the chronic human Trypanosoma cruzi infections with nitroderivatives did not lead to parasitological cure. Seventeen treated and 17 untreated chronic Chagas' disease patients, with at least two out of three positive serologic assays for the infection, and 17 control subjects formed the study groups. PCR assays with nested sets of $T$. cruzi DNA primers monitored the efficacy of the treatment. The amplification products were hybridized to their complementary internal sequences. Untreated and treated Chagas' disease patients yielded PCR amplification products with $T$. cruzi nuclear DNA primers. Competitive PCR was conducted to determine the quantity of parasites in the blood and revealed $<1$ to $75 \mathrm{~T}$. cruzi $/ \mathrm{ml}$ in untreated (means $25.83 \pm 26.32$ ) and $<1$ to $36 \mathrm{~T}$. cruzi/ml in treated $(6.45 \pm 9.28$ ) Chagas' disease patients. The difference between the means was not statistically significant. The findings reveal a need for precise definition of the role of treatment of chronic Chagas' disease patients with nitrofuran and nitroimidazole compounds.

\section{COMMENTARIES}

The use of a molecular method to evaluate the results of chemotherapy of Chagas disease is welcome and the authors are to be praised for their work. From the technological viewpoint, the study is of the highest possible quality, reporting, for the first time, the quantitative polymerase chain reaction (PCR) to detect subclinical parasitemias in 34 patients with chronic Chagas disease.

Unfortunately, however, the results shown do not support the conclusions drawn.

In an article referred to by the authors ${ }^{2}$, we have clearly demonstrated that quantitative conventional serological tests, i. e., complement fixation $(\mathrm{CF})$, indirect immunofluorescence (IF), indirect hemagglutination (HA) and enzyme-linked immunosorbent assay (ELISA), represent an entirely reliable basis for the criterion of cure of American trypanosomiasis. When positive, these tests conclusively demonstrate current $T$. cruzi infection and the presence of the parasite in the patient tissues. Conversely the post-therapeutic persistent negativity of these quantitative serological tests demonstrate cure.

In the summary transcribed above ${ }^{1}$, three study groups were utilized, comprising 51 male street-cleaners in Brasília: 1) seventeen treated; 2) seventeen untreated and 3) seventeen controls, who had serological tests negative for T. cruzi infection. Both the 17 treated and 17 untreated patients had "at least two out of three positive serologic assays for the infection". Therefore, the group of 17 treated patients (who were treated 10 years earlier, by different physicians at the Medical Offices of the Brasília Refuse Department) consisted of patients who had not been cured, as shown by their positive conventional serological tests. So it is clear that both the treated and the untreated groups are equal, consisting of patients with active Chagas disease. Thus, it was to be expected that PCR would reveal subclinical parasitemias in these individuals. The fundamental confusion is that treated patients are not cured patients. Consequently, there is no basis for the assertions that nitroderivatives do not lead to parasitological cure or that assessment of cure after treatment of acute and chronic Chagas disease requires a highly sensitive molecular method for detecting subclinical parasitemias. 


\section{THE REAL CONTRIBUTION}

Finally, though the results do not sustain the proclaimed ideas, this pioneer investigation is not without merit. On the contrary, it is a significant contribution. By detecting subclinical parasitemias in 34 patients with long standing chronic Chagas disease it adds further proof that positive conventional serological tests reflect the presence of $T$. cruzi in the patient's tissues, supporting the concept that pathogenesis of the disease depends on the presence of the parasite.

\section{REFERENCES}

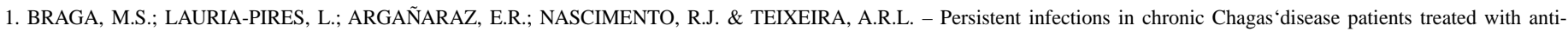
Trypanosoma cruzi nitroderivatives. Rev. Inst. Med. trop S. Paulo, 42: 157-161, 2000.

2. CANÇADO, J.R. - Criteria of Chagas disease cure. Mem. Inst. Oswaldo Cruz, 94(supl. 1): 331-335, 1999. 